\section{DEATHS AFTER OVARIOTOMY, WITH REMARKS ON THE VALUE OF FLUSHING WITHOUT SUBSE- QUENT DRAINAGE OF THE PERITONEAL CAVITY.}

BY W. A. MEREDiTH, M.B. EdiN., F.R C.S. ENG., SENIOR SURGEON TO THE SAMABITAN FREE HOSPITAT.

Is October, 1888, I read before the Rogal Medical and Chirurgical Society a paper on the "Mortality of Abdominal section," founded chiefly upon the results of a first series of 100 ovariotomies, of which number $10 \mathrm{had}$ proved fatal.: In February of the present year I completed a further series of 250 operations for the removal of ovarian tumours, with 243 recoveries and but 7 deaths - a mortality of 28 per cent. The following details of these 7 fatal cases appear to me to be worth recording as illustrative of some of the risks and complications liable to be encountered in dealing with ovarian growths; and I venture to hope that this relation of my failures may prove of some use to others who are working in the same field as myself.

CASE 1 (145). ¿-A woman, aged fifty-six years, the mother of four children, was sent to me at the Samaritan Hospital in October, 1890, by Dr. Livingston, of Hursley. The menopause had occurred seven years before, and during this interval she had once seen a return of coloured discharge five years previously to her admission. She was greatly emaciated and both lower limbs were very cedematons ; the abdomen was enormously distended by a cystic tumour displacing forwards the xiphoid cartilage, and expanding the costal arch on each side. On Oct. 24th double ovariotomy was performed. The left tumour-a practically unilocular cystwas completely sessile, necessitating for its removal a very extensive and troublesome enucleation; the resulting rent in the left broad ligament was subsequently closed by a series of six deeply-placed interlocking ligatures for the arrest of hrmorrhage, and the peritoneal edges were then accurately united by a continuous suture of fine silk. The right tumour, consisting of a thick-walled very multilocular growth with a good deal of semi-solid material, was unadherent, and its removal entailed no difficulty. The abdomen was closed without flushing or drainage, the relaxed parietes being carefully supported by strapping and a flannel binder applied over the usual dressings augmented by a large mass of cotton wool. The aggregate weight of the tumours exceeded $50 \mathrm{lb}$. (42 pints of flaid and nearly $9 \mathrm{lb}$. of solid material). Convalescence during the first week was quite uneventful, the highest recorded temperature being $99^{\circ} \mathrm{F}$., with a pulserate of 76 ; the bowels acted naturally from the third day onwards; and the dressings were changed on the sixth day, when the incision was found to be well united, and the sutures were consequently removed, the abdomen being subsequently carefully restrapped and supported as before by a binder. During the next forty-eight hours progress continued to be satisfactory, and on the evening of the eighth day the patient was left in excellent spirits, with a perfectly normal temperature and pulse, without cough or discomfort of any kind, and apparently well advanced on the road to complete recovery. The weather at the time was exceedingly wintry, with a biting east wind and very low temperature, and during the night she must in some way have been exposed to chill, for at my visit next morning I found her condition totally altered. She was lying propped up in bed complaining of severe dyspncea and of pain in the left side of chest, examination of which revealed some deficiency of resonance over the base of the lung with much coarse crepitation; the left upper lobe and the right lung tbroughout were resonant, but the breath sounds were much obscured by rîles. The abdomen was quite soft and natural, the bowels having acted without help during the early morning ; per vaginam the uterus was found freely mobile, and no tenderness or fulness was detected in the pelvis. The temperatare and the

I Transactions of the Royal Medical and Chirurgical Society for 1889, vol, Ixxii

2 The figures in parentheses refer to the total number of ovariotomiea performed. pulse, which were $98.4^{\circ}$ and 72 respectively on the previous evening, had risen to $102 \cdot 2^{\circ}$ and 116 respectively; the respirations were 36 , and there were no sputa, although cough was constant. During the next twenty-four hours the temperature rose gradually to $104^{\circ}$, with a very feeble, intermittent pulse, and a moderate amount of thin, frothy, muco.purvlent secretion was brought up, but expectoration was evidently much impeded by the relaxed state of the diaphragm and abdominal muscles. The patient rapidly lost ground in spite of very free stimulation, the breathing becoming more and more laboured and the heart's action more enfeebled as the lung secretion accumulated, until death took place on the morning of the twelfth day, seventy-two hours subsequently to the onset of the lung mischief. I was unable to obtain a post-mortem examination, but there could not under the circumstances be any doubt as to the cause of death.

This unfortunate termination to a promising case was very disappointing, although on reviewing it I cannot see how recovery from such a condition could well be hoped for. The lesson to be learned from it points to the advisability, when dealing with large, old-standing ovarian cysts which exert much upward pressure on the diaphragm, of removing a portion of their fluid contents by aspiration a few days before the major operation is undertaken; by so doing one may promote the gradual expansion of the compressed lower pulmonary lobes, and thus place the patient in a better condition for withstanding the shock of a severe operation and for subsequently resisting the onset of any lung complication should such arise.

CASE 2 (212).-A single woman, fifty-eight years of age, of feeble physique and extremely emaciated, was admitted into the hospital in April, 1893. The menopause had occurred five years before, and she had known of the existence of an abdominal tumour for about a year. Four months previously to admission she had suffered from a sudden attack of abdominal pain, which confined her to bed for seven weeks. On examination the abdomen was found to contain an extremely tender, elastic tumour extending upwards on the left side to the level of the costal arch, and apparently fixed by adhesions. The history suggested a twisted pedicle. The patient was operated upon on April 25th. The tumour proved to be an ovarian cyst covered by coils of adherent intestine and connected below with the right side of a normal uterus by a very tightly twisted pedicle showing three distinct turns. The cyst wall was very rotten, of a dark-greenish hue over its upper portion, and dark purple in colour towards the base, where it was extensively adherent in the pelvis; the contained fluid measured 9 pints. The pedicle was trans fixed and tied well below the lowest twist, and before closure the abdominal cavity was flushed out and a drainage-tube inserted. The operation, performed at 9.30 A.Mr., lasted just. over one hour, and the patient soon regained conscious ness. During the first twelve hours the condition was fairly satisfactory, the temperature rising slowly to $100^{\circ} \mathrm{F}$. with a somewhat feeble pulse of 110 . At 9 P.M. the tube dressings were changed, about $\frac{1}{2}$ oz. of blood-stained fluid being withdrawn; at the same time 3 oz. of urine were obtained by the catheter, of low specific gravity and containing a small amount of albumin, of which no trace had been discovered before operation. Shortly after this the patient became restless and the temperature rose to $102^{\circ}$, with a very rapid intermitting pulse; no more urine was secreted and she gradually became insensible, dying quietly at 4 A.M., about seventeen hours after the removal of the tumour.

The post-mortem examination revealed nothing amiss in the peritoneal cavity; both kidneys were contracted and granular with very adherent capsules; the heart, weighing $9 \frac{1}{2}$ oz., was laden with fat and its substance was soft and flabby, the cavities were distended, and the valves normal. The cause of death was attributed to shock consequent on cardiac and renal disease of old standing.

CASE 3 (250).-A very feeble and emaciated woman, aged sixty-six years, was first seen in October, 1894, in consultation with Dr. Uhthoff, of Brighton. The menopanse had occurred at the age of fifty-one years, but for ten years previously to the date of my visit she had been subject to occasional slight uterine hæmorrhages, although her general health had continued fairly good until about twelve months before (October, 1893), when she began to lose flesh rapidly and the abdomen enlarged. In May, 1894, she consulted Dr. Uhthoff, who tapped her, removing 25 pints of (?) free fluid. This operation was repeated in the following Jaly, when $12 \frac{1}{2}$ pints were taken away. At my examination three months later $I$ found a considerable quantity of 
peritoneal fluid surrounding an elastic tumour, of which the base entirely filled the pelvic cavity, displacing upwards a bulky uterus, which was easily recognisable above the pubes in close connexion with the anterior surface of the tamour. The bowels were evidently much loaded; the urine was scanty, highly coloured, and of specific gravity 1016; there was no albumin. My diagnosis was ovarian tumour, possibly malignant, with ascites, and I advised exploration. The operation was performed on Oct. 20th, 1894. The growth proved to be a semi-solid ovarian tumour composed mainly of brain-like material of extremely soft consistence and very suggestive of malignancy. ${ }^{3}$ Adhesions were very extensive, especially in the pelvis, where a broad vascular pedicle was connected with the right side of the uterus. The left Fallopian tube was distended to the size of a large sausage (hydrosalpinx). I tapped, but did not remove it, as the corresponding ovary was quite atrophied. Finally, the abdominal cavity was well flushed and closed without drainage. The weight of the tumour, apart from the ascitic fluid, amounted to between $9 \mathrm{lb}$. and $10 \mathrm{lb}$. During the first five days progress was uneventful, with a temperature not exceeding $99.2^{\circ} \mathrm{F}$, and a pulse-rate varying between 90 and 100 ; nourishment was well taken, the kidneys acted freely, and the abdominal condition remained satisfactory; the only trouble complained of was want of sleep. On the morning of the fifth day the bowels acted with the help of an enema, dark liquid motions with numerous small scybala, and these recurred at frequent intervals throughout the day, accompanied by the escape of large quantities of extremely offensive flatus. The patient complained for the first time of very severe headache, with a general sense of depression, and towards evening she was inclined to wander. This mental condition became aggravated during the night ; she grew very restless, requiring some control to prevent her from getting ont of bed, and constantly complaining of the pain in her head; there was no sickness, and she took plenty of liquid nourishment; the temperature touched $100^{\circ}$ for the first time since the operation, with a pulse of 108 . When seen in the morning of the sixth day she appeared so worn out by want of sleep that Th administered one-third of a grain of morphia hypodermically. Shortly after that she fell asleep, and remained so throughout the afternoon, bresthing very deeply and irregularly at the rate of only 12 respirations per minute; the face was very flushed, the eyelids were only partially closed, and the pupils moderately contracted; the temperature varied between $99.8^{\circ}$ and $100^{\circ}$, with a pulse-rate of from 72 to 96 . She took nourishment readily without fully awaking and passed urine at rather frequent intervals. At 9.30 P.M. after she had remained in this condition for between eight and nine hours, I roused her without any diffculty, but found her quite as excitable and wandering as before her sleep. She still complained greatly of headache, and was extremely restless until after the bowels had again acted at 11.30 P.M., when she grew quieter, the temperature meantime having risen to $101^{\circ}$, with a pulse of 116 . She gradually passed into a comatose condition, and so remained until her death at 5 P.M. on the following day, which was preceded by two distinct convulsive seizures, each one lasting for about a minute.

I was unable to obtain leave for an examination, and therefore cannot be certain as to the cause of death, but judging by the onset and development of the fatal symptoms I am inclined to attribute them to toxic poisoning from intestinal absorption in an aged and very feeble subject with unhealthy kidneys. The advisability of giving morphia in such a case is more than doubtful, and I certainly should not again resort to it under similar circumstances, although $I$ hardly think that in the present instance its administration materially influenced the result.

CASE 4 (275).-A woman, aged seventy years, was first seen in consultation with Dr. Gray, of Oxford, and $\mathrm{Mr}$ Garnham, of Herne-hill, in January, 1895. The abdomen contained a very mobile ovarian cyst of moderate size, and I advised early operation in view of the likelihood of the pedicle becoming twisted. Shortly after my visit the patient was laid up by a sharp attack of influenza, subsequently complicated by bronchitis, and operative treatment was con sequently postponed for some months. I did not again see her till the following June, when the cyst appeared to me to be less freely moveable and decidedly more tender than before,

3 The specimen, reserved for microscopical examination, was unfortunately thrown away. but I failed to obtain any definite history pointing to rotation of the pedicle. On June 7th, 1895, the operation was performed. The tumour proved to be a left ovarian cyst, from 7 to $8 \mathrm{lb}$. in weight, with a very tightly twisted pedicle and universal adhesions. Its removal was a very troublesome business, necessitating the application of numerous ligatures to omental, intestinal, and mesenteric connexions. The abdominal cavity was freely flushed out at the conclusion of the operation and closed withont drainage. During the first five days all went on well. The temperature once touched $100 \cdot 6^{\circ}$ with a pulse of 88 in the evening of the second day, but fell to normal in the course of the next twelve hours and did not rise again. Nourishment was well taken after the first twenty-four hours, and the abdomen remained free from distension or tenderness; the kidneys acted abundantly from the first. At $2 \mathrm{~A} . \mathrm{M}$. on the sixth day after the operation the patient woke up suddenly complaining of stomach ache, and shortly afterwards vomited some coffee-ground fluid; an hour later a small dark blood-clot was passed per anum, which was presumed to proceed from hæmorrhoids, and a soap-and-water enema, administered to promote an action of the bowel, returned almost immediately mixed with a quantity of dark fluid blood without trace of fæces. I was at once sent for, and on arriving at $5 \mathrm{~A}$ M. found the patient looking pale and anxious and inclined to be somewhat restiess, although quite free from pain; the abdomen was soft and not notably tender anywhere on pressure, and normally resonant, without a trace of distension; the temperature was $97 \cdot 8^{\circ}$, with a compressible pulse of 84 . Nothing was to be felt per rectum, and I came to the conclusion that the hæmorrhage must proceed from a stercoral ulcer. At 6 A.M. a further considerable quantity of dark fluid blood was voided per anum, and this same recurred at 7.30 A. M., shortly before which hour some coffee-ground fluid was again vomited. The temperature had now fallen to $97^{\circ}$, while the pulse had risen to 120 . No more blood was passed until 9.15 A.M., when a further very copious discharge took place, after which the patient rapidly collapsed and died quietly at 9.45 A.M., within eight hours of the first manifestation of her condition. The post-mortem examination, made by Dr. Allchin, revealed no trace of peritonitis ; the pelvic cavity contained two ounces of blood-stained fluid. All the tissues were markedly exsanguine. The liver, spleen, and pancreas were fairly normal, but both kidneys were small, and on section showed a very advanced condition of interstitial nephritis. The lungs were healthy, but the heart was enlarged and loaded with fat. The intestinal canal throughout showed deep purple staining of the mucosa, which was otherwise normal until within one and a half inches of the pylorus, where an irregular-shaped ulcer was discovered, evidently on the verge of perforation, and adhering to its floor was seen a small, recent blood-clot seated in close proximity to what appeared to be an ulcerated ressel. To quote Dr. Allchin's report: "I have no doubt from the general appearances that the hæmorrnage occurred from this site. The duodenal ulcer was an old one-i.e., of several years' standing. Death is amply accounted for by the hæmorrhage, which must have been very considerable, as the bloodvessels generally were quite empty. The condition of the kidneys, however, was such that I much doubt whether the patient could in any event have recovered."

This case is, I believe, unique in the annals of ovariotomy, so far as regards the cause of death. The absence of any premonitory symptoms whence the existence of a duodenal ulcer might have been deduced rendered it impossible to accurately locate the source of the hæmorrhage when the patient was first seen on the morning of her death; and although it seemed clear enough at the time that the bleeding must proceed from a stercoral ulcer I pictured this as probably situated in the large bowel. Under the circumstances, however, no operative treatment could have possibly saved her, even though undertaken with accurate knowledge as to the seat of the bowel lesion.

CASE 5 (304).--The patient; a young woman, nineteen years of age, first seen in consultation on March 17th, 1896, had apparently enjoyed good health until fire days before, when, coincidently with the onset of the monthly period, she was suddenly seized with very severe abdominal pain, accompanied by vomiting and fever. On examination under an anæsthetic $I$ found the abdomen occupied by a tense elastic swelling, rising to just above the level of the umbilicus and filling the pelvic cavity below in front of a small uterus, which was displaced backwards into the right posterior 
quarter of the space. I considered the tumour to be ovarian, probably with a recently twisted pedicle; but as men. struation was persisting very freely at the time it was decided to postpone interference for a few days. During the course of the next forty-eight hours the flow gradually lessened and both pain and sickness were much relieved; the temperature, from $101^{\circ} \mathrm{F}$., subsided to $996^{\circ}$, and the pulse, which had been extremely feeble and rapid, slowed down to about 100 . Operation was performed at $11 \mathrm{~A}$ M. on March 22ad. On exposure the cyst wall appeared of a deep plum colour. After separation of extensive recent parietal, omental, and pelvic adhesions the tumour was tapped and four pints of ordinary ovarian fluid, but slightly blood-stained, were withdrawn, and the flaccid sac which, although very soft and friable, was tolerably thick-walled, was then extracted whole without difficulty. The pedicle connected with the right side of a normal uterus was unusually short and had rotated one full half turn from left to right; it was secured by transfixion below the twisted portion previously to division. The peritoneal cavity was then flushed and closed without drainage. I saw the patient again at 6 P M., when her condition appeared to be quite satisfactory; the temperature was $998^{\circ}$ and the pulse 108 ; the skin was moist; there were no sickness and no unusual amount of pain; the kidneys had acted freely, and she expressed herself as feeling much more comfortable than she had expected. I heard nothing more until eight o'clock the next morning, when a telegram reached me stating that she had died at $6 \mathrm{~A} M$. On inquiry I learned that in the course of the previous evening she became somewhat restless, the temperature rising to $102^{\circ}$, with a pulse of 120 ; but her condition apparently gave no cause for alarm when she was visited by her medical attendant at 10 P.M., as there were no other unpleasant symptoms of any kind. During the next five hours, however, the restlessness increased, as the temperature gradually mounted to $103^{\circ}$; by $4 \mathrm{~A} \mathrm{M}$. it had reached $104^{\circ}$, and the stage of excitement had passed on to actual delirium. At 4.45 A M. a dose of morphia was administered hypodermically, and this was repeated balf an hour later. Shortly after the second dose the patient became comatose and died quietly at 6 A.M., with an ante-mortem temperature of $1054^{\circ}$.

As no examination was permitted one cannot feel certain as to the cause of death; but on reviewing my notes I am inclined to attribute it to hyperpyrexia induced by shock in an extremely nervous and excitable patient. The administration of morphia under the circumstances was a mistake. The proper treatment would bave been to reduce the temperature at any cost, and this should have been effected by the continuous application of ice to the head, combined with cold wet packing of the extremities.

CAsE 6 (314).-A married woman, aged forty-eight years, anxious-looking, spare, and very anæmic, the subject of a moderate-sized goitre, was admitted into hospital under my care at the request of Dr. Raby, of Devizes, in May, 1896. Eight years previously to her admission she noticed a small lump situated in the left iliac fossa, which was diagnosed as a uterine fibroid. The tumour grew very slowly until about nine months before I saw her, when it suddenly took on rapid increase. She was extremely emaciated, and stated that she had persistently lost flesh ever since a severe attack of diphtheria five yeais before. The menopause dated three years back. The urine was somewhat scanty and of low specific gravity (1015); no albumin was discoverable. The abdomen was filled by a tense cystic tumour rising to the level of the costal arch and extending deeply in the pelvis, whence the uterus was displaced upwards into the abdominal cavity, being there recognisable in close connexion with the front of the tumour somewhat to the left of the middle line. Operation was performed on May 28th, 1896. The tumour proved to be a double fused ovarian cyst containing fourteen pints of flaid laden with cholesterine. Its upper portion was unadherent, but below the level of the umbilicus its relations became very complicated; in the middle line the cyst wall was intimately connected with the posterior surface of the displaced uterus, whence the growth burrowed deeply on either side between the layers of the broad ligaments, being overlaid by the Fallopian tubes, both of which were greatly hypertrophied. The deeper portions of the tumour were very firmly embedded on the left side beneath the sigmoid flexure and upper two-thirds of the rectum, and on the right beneath and behind the cæcum, whence the vermiform appendix, thickened to the size of one's little finger, and elongated to the extent of seven or eight inches, overlay the growth, and was firmly adherent to the fundus uteri. The enucleation of these deeper portions was an exceedingly difficult and tedious business, owing to the toughness of the attachment and the close connexion with bowel on both sides; but it was at last satisfactorily accomplished. The very firm adhesion to the back of the uterus was cautionsly severed by means of scissors, and finally both ovarian pedicles were ligatured and divided. The numerous pressure forceps were then removed, and the peritoneal cavity having been very freely flushed out was closed without drainage. The operation lasted two and a half hours. The patient soon recovered consciousness; the temperature, which was $98.2^{\circ} \mathrm{F}$. when she was placed in bed, rose to $100 \cdot 8^{\circ}$ in the course of the next few hours, with a pulse of 120 , about the same rate as before the operation. During. the next twenty-four hours the temperature ranged between $100^{\circ}$ and $101^{\circ}$, but the pulse beats mounted to 140 ; there was no sickness, and flatus escaped very freely, so much so as to interfere with the retention of nutrient enemata: the kidneys acted abundantly. On the second day the average temperature was somewhat lower, but the pulse rate increased, varying between 144 and 160; the bowels acted freely several times, and the rectal injections were subsequently better retained. During the night the patient had a few hours' sleep for the first time since the operation, and awoke somewhat refreshed, but there was no material alteration in her condition during the third day, excepting that she showed aversion to taking nourishment, which during the previous forty-eight hours she had accepted readily. Early in the morning of the fourth day she became very restless and excitable, although she had slept at intervals during the night; the temperature, which in the previous day had fallen to below $100^{\circ}$, rose to $102^{\circ}$, with a pulse of 140 ; the bowels acted freely with a soap-and-water enema, which was repeated in the course of the day. Her mental condition, however, continued very unsatisfactory, alternating between semi.maniacal attacks, during which she required restraint, and complete lapses of consciousness lasting for an hour or two hours at a time. She obstinately refused to swallow any nourishment, and voluntarily expelled the nutrient enemata and suppositories, the administration of which was rendered extremely difficult by her violence. After lingering on in this hopeless state for seventy-two hours she died practically from starvation early on the morning of the eighth day after the operation, no nourishment of any kind having been swallowed or retained during the last four days of life.

The following report of the post-mortem examination was furnished to me by Dr. Allchin. " Rigor mortis well marked body extremely emaciated; no attempt at union of the incision; abdominal cavity perfectly dry, without trace of peritonitis anywhere; the hollow viscera were extremely retracted and there was a very notable absence of fat. All abdominal organs were healthy with exception of the kidneys, of which the right one was small and moderately contracted, while the left was converted into a mere sac owing to dilatation of pelvis and calyces, the renal substance being reduced to a thin shell barely a quarter of an inch in thickness; no actual obstruction of the left ureter was dis. covered, and it must therefore be presumed that the con. dition resulted from long-standing pressure (i.e., of the tumour in the left side of the pelvis)." The cause of death was entered as inanition, renal inadequacy being set down as a secondary factor.

This is the only fatal case of insanity which I have met with in my abdominal work. In but two other instances (one ovariotomy and one abdominal hysterectomy) have I seen well-marked mental trouble following upon the removal of large abdominal tumours, and both these patients recovered completely within a few months of the operation. The above three cases are the only ones met with in a series of over 700 abdominal sections, and in each instance the tumour had existed for many years before its removal-a fact worth noting with regard to prognosis under such circumstances. On the other hand, a slight degree of mental disturbance, usually characterised by moroseness, with occasional fits of irritability, is not an extremely uncommon feature of early convalescence after removal of large tumours in middle-aged women; this condition in my experience is a merely tran. sitory one, most noticeable in patients who have long suffered from chronic constipation associated with the presence of slowly growing pelvic tumours.

CASE 7 (349).-A stout and very anæmic woman, aged forty years, the mother of one child twenty-two years old, was admitted into hospital in January, 1897, suffering from 
pelvic pain of a sufficiently severe character to incapacitate her for work. She had been in failing health for some eighteen months, and had latterly begun to lose flesh. The heart-sounds were feeble, but no murmur was detected; the urine was scanty, highly coloured, and slightly turbid, of specific gravity 1025, and there was no albumin. The abdominal parietes were loaded with fat, excepting over the seat of a large, irregular, depressed cicatrix in the right hypochondrium, said to have been caused by the bursting of an internal abscess twenty years before. The left iliac region was occupied by a firm, tense swelling rising out of the polvis to the level of the crest of the ilium, and extending just across the middle line. Per vaginam the base of the tumour filled the left half of the pelvic cavity, being evidently closely connected with the left side of a somewhat bulky uterus. The mass was diagnosed as an intraligamentous ovarian cyst. Operation was performed on Feb. 10th, 1897. The abdominal wall contained a most unusual amount of fat, and the subjacent omentum was adherent to the parietal peritoneum. The right ovary, cystic and enlarged to the size of a hen's egg, was buried in old adhesions at the bottom of the pelvis, and its removal entailed a good deal of difficulty; the Fallopian tube overlying it contained a mass of cretaceous material-presumably the remains of an old pyosalpinx. The tumour on the left side, of the size of an infant's head, proved to bs a sessile ovarian cyst completely encapsuled between the layers of the broad ligament, and overlaid externally by the sigmoid slexure. Its enucleation was a troublesome business owing to the extreme toughness of its connexions and the depth to which it extended; the contained fluid was laden with cholesterin. The damage inflicted on the broad ligament during the removal of the growth precluded the possibility of successfully suturing the remains of the torn capsule. I therefore freely flushed out the abdominal cavity and closed it at once. For the first $t$ wenty-four hours after the operation the patient's condition seemed satisfactory, excepting for the fact of an extremely feeble circulation. During the second night her temperature rose slowly to $101 \cdot 6^{\circ} \mathrm{F}$., with a pulse of 114, and both remained at or about this level for the next twenty-four hours, during which there was no sickness, the flatus passed regularly, and the kidneys acted fairly well. On the morning of the third day it was noted that the pulserate had increased to between 130 and 140 , the temperature meanwhile having risen to $1024^{\circ}$; there was no inclination to romit, but the flatus had ceased passing, and the urine, which had become scanty, contained albumin. Towards evening the abdomen became very distended at the upper part, more especially in the left hypochondrium; a turpentine enema, administered through a long tube passed without difficulty for some eight or nine inches up the bowel, was followed by a very free escape of flatus, which afforded much relief for a time; but the distension recurred together by extremely tough, fibrous adhesions of very old standing, of such density as to prove inseparable except by the use of the knife; the liver and spleen were further firmly adherent to the diaphragm, and the former was also closely attached to the abdominal wall down to the level of the scar in the right hypochondrium; the omentum was firmly united to the underlying intestinal coils, which were matted together by old adhesions. Both kidneys were small and pale, with very adherent capsules. The heart was laden with fat and its substance was pale and fatty; the lungs were fairly healthy ; all the tissues were extremely anæmic. The pelvic pouch contained two or three ounces of blood-stained fluid; both ovarian pedicles appeared healthy; the uterus was bulky and its cavity contained a submucous fibro-myoma of the size of a duck's egg. The state of the abdominal viscera here described was amply sufficient to account for the fatal result when viewed in connexion with the condition of the heart and kidneys. There can be little doubt but that the adhesions dated from the attack which led to the discharge of pus through the abdominal wall twenty years before my operation; and the fact of the patient's survival and enjoyment of comparative good health during so many subsequent years, in spite of the existence of such a complicated condition of the abdominal viscera is by no means the least interesting feature of the case.

Remarks.-The fact of 243 recoveries in a series of 250 major operations is satisfactory, but the comparative ease or difiticulty of the individual recoveries is also worth some consideration. In the investigation of this subject a careful examination has been made of the notebooks of the case in question, each one of which contains a complete record of the axillary temperature taken every two hours during the first two days after operation, and subsequently every four hours until the end of the first week, when the entries become less frequent as convalescence progresses. For purposes of classification I have selected in each case the highest temperature noted during the patients' stay under $m y$ care, although such record in the great majority of instances has been reached on but one, or at most two, occasions during the first forty-eight hours after the operation. By this method I have arrived at the results given in the subjoined table, wbich shows, in addition, the number of cases where special means were adopted for cleansing the peritoneal cavity, consisting of drainage (D.), of flushing followed by drainage (E.D.), or finally of flushing with immediate closure of the abdominal incision (F.) ${ }^{4}$ The grouping of the cases in consecutive series of 50 serves to indicate the gradual adoption of the last:named plan of treatment as a substitute for either of the two former methods. The classification of the temperature records is that given in the article on Temperature contained in the last edition of Quain's Dictionary of Medicine.

Two Hundred and Fifty Ovariotomies with Shven Deaths.

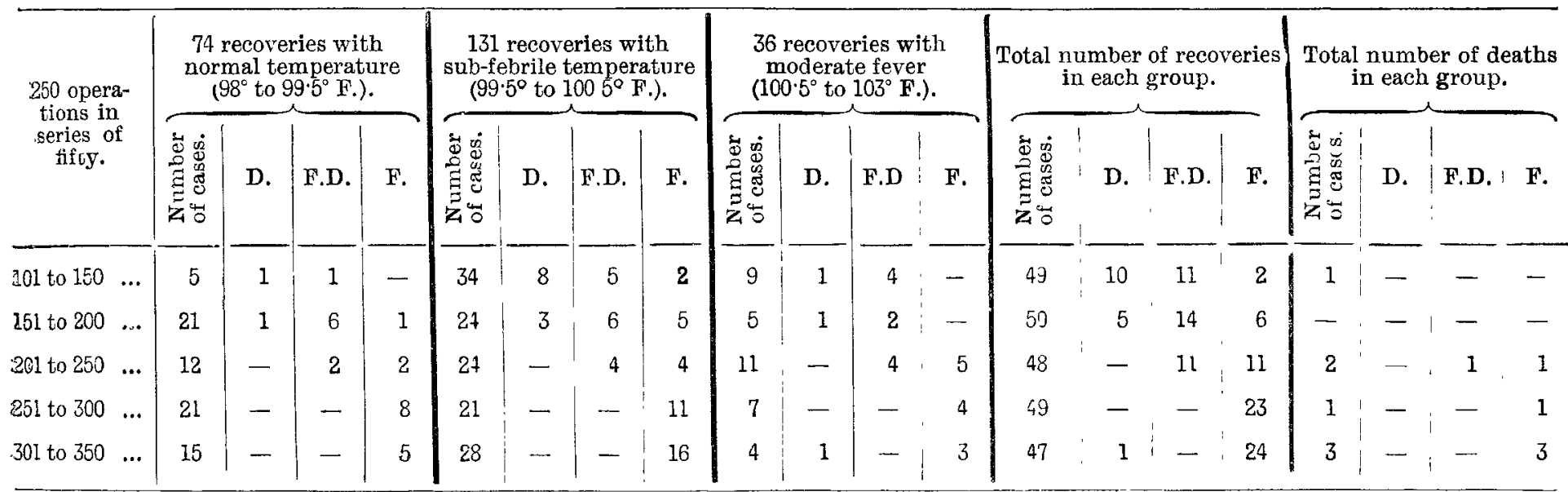

Two recoveries with high fever (i.e., exceeding $103^{\circ} \mathrm{F}$.) complete the total of 243 . One of these cases occurred in the first group and the other in the third; both were flushed and drained.

during the night and a repetition of the $\epsilon$ nema on the following morning produced no such result, and was succeeded by the vomiting of some coffee-ground fluid. From this time the patient rapidly lost ground, the heart's action becoming more and more feeble, as the distension increased, until her death, which took place before the completion of the fourth day. Post-mortem examination revealed no trace of recent peritonitis, but the following state of things was discovered the liver, spleen, stomach, and transverse colon were matted
It will be seen that in 205 cases ( 84 per cent. of the total number of reccveries) the temperature never exceeded $100.5^{\circ} \mathrm{F}$., which point may, I think, be taken as the limit for what should be considered as a normal convalescence after a major abdominal operation; of the remaining 38 cases, 36

4 The term flushing, as used in this connexion, is preferable to the word irrigation, which, although correctly epplicable in the case of ordinary surgical wounds, is not accurately descriptive of the process of washing out the peritoneal cavitr. 
recovered with a moderate amount of fever (not exceeding $101.5^{\circ}$ in 24 instances); and in only 2 cases out of the total number of 243 recoveries was there a rise of temperature sufficient to constitute high fever.

Special means for cleansing the peritoneal cavity were adopted in 118 (over 48 per cent.) of the 243 successful cases in the following relative proportions: Drainage in 16 instances-about 1 in 15 ; flushing and drainage in 36 instances-about 1 in 7 ; and flushing in 66 instances-about 1 in 4 . To the adoption of this last-named method of treatment in cases presenting extensive adhesions or other intra-peritoneal complications 1 am inclined in great measure to attribute the results obtained, not only as regards actual recovery, but also in respect to the comparative ease and shortness of convalescence, and I therefore think it worthy of brief consideration here.

The value of flushing as a convenient and efficient means of removing blood-clot or effused fluids from the peritoneal cavity has now been pretty generally recognised for some years past, the usual practice in this respect being, so far as I am aware, to subsequently remove as much as possible of the water thus introduced, and, further, to provide, by the insertion of a drainage-tube, for the escape of any remainder which might not be readily absorbed. That such absorption takes place very rapidly, however, during the first twentyfour hours after operation is amply evidenced by the comparatively small amount of fluid to be obtained through the tube in cases where a very considerable quantity has been left in the abdomen, and it was the observation of this fact that first led me to try the effect of leaving the closed peritoneal cavity filled with water in the expectation that the serous membrane would be able safely to dispose of this fluid, together with any remaining bloodclot or any other concrete substance which by solution might be rendered absorbable. The results following this treatment, which I first carried out in a case of ruptured colloid ovarian cyst with very extensive peritoneal infection in March, 1889, have fully answered my expectations. Of the 66 cases above mentioned in which the closed abdominal cavity was designedly left filled with water at the conclusion of the operation, ${ }^{5} 16$ recovered with a normal temperaturei.e., not exceeding $99.5^{\circ}$; 38 recovered with a sub-febrile temperature, varying between $995^{\circ}$ and $100.5^{\circ}$; and 12 recovered with moderate fever, ranging in six instances between $1005^{\circ}$ and $101.5^{\circ}$, and in the remainder between $101.5^{\circ}$ and $103^{\circ}$

These results appear to me sufficient to warrant one in advocating a method of treatment which has certainly helped in promoting them, and I will therefore, in conclusion, briefly enumerate what I consider to be the advantages attainable by its adoption. 1. The enclosed water, while tending in some measure to arrest venous oozing by the hæmostatic action due to its temperature, further acts beneficially by preventing fresh formation of clot in the event of the temporary persistence of such oozing after closure of the abdomen 2. By its solvent action on any concrete substances, such as blood-clot, colloid material, or lymph, which may have escaped removal during the process of washing out, it promotes their absorption and thus prevents the establishment of a nidus for the development of septic mischief. 3. In a similar manner, by dilition, it facilitates the absorption of any ovarian or other remaining fluids liable to provide a medium for the growth of micrococci. 4. Owing to the rapid absorption of fluid thus taking place from the peritoneal cavity during the forty-eight hours immediately succeeding operation the urinary secretion is freely promoted from the first-a fact of considerable importance in cases of renal inadequacy, whether this condition be dependent on a temporary cause such as antecedent congestion resulting from pressure, or be due to one or other of the chronic forms of renal disease so frequently to be met with in middle-aged patients. 5. Viewed as a mechanical agent, the enclosed fluid by its mere bulk assists in restoring the balance of intra-peritoneal pressure, disturbed by the removal of a large tumour, and hereby promotes recovery from shock in cases of this nature. 6 . As a result of natural gravitation, the water, by displacing the intestinal coils from the pelvic cavity, certainly tends to prevent the formation of adhesions in this situation. 7 . Finally, by affording a liquid medinm throughout the peritoneal cavity, it diminishes friction and consequent irritation

3 The hot water used for this purpose bas in everg instance been obtained from the usual domestic boiler supplv, its temperature bein
regulated when necessary by the addition of ordinary cold tap water. between opposed serous surfaces, intestinal or other, during: the first twenty-four hours or so after operation, thereby lessening pain, averting the risk of adhesions, and generally promoting ease and smoothness of recovery.

In conclusion, I may state that my views regarding the value of the treatment in question are founded, not only upon the cases here dealt with, but, further, upon the experience of equally satisfactory results obtained in numerous operations. for various abdominal conditions other than those now under consideration. It is hardly necessary to point out that the immediate closure of the abdominal cavity here advocated is, of course, contra-indicated in cases complicated by antsesedent. intra-peritoneal sepsis at the time of operation.

Manchester-square, $\mathrm{W}$.

\section{ON THE PATHOLOGY OF RECURRENT APPENDICIT'TS. 1}

By F. A. SOUTHAM, M.B. OxON., F.R.C.S. ENG.,

SURGEON TO THE MANCHESTER ROYAL INFIRMARY, AFD MHCTURER ONS OPERATIVE SURGERY, OWENS COLLHGE.

OUR knowledge of the pathology of recurrent appendicitis until quite recently was very imperfect, but it is now much more complete since it has become the custom to employ operative measures for its relief, for opportunities are offered us of studying the changes which take place in the appendix at an early stage in the course of the disease. The following remarks are based on the conditions which were found to be present in twenty cases treated by operation, details of which are given in the accompanying table. Of the 20 cases, 7 occurred in females and 13 in males. The youngest patient was ten and the oldest was forty-four years of age. In 15 of the cases the patients were between fifteen and thirty years of age. In all the specimens examined the appendiz showed eridences of chronic inflammatory changes, its coats - mucous, muscular, and peritoneal - being all thickened. In some cases its lumen was uniformly narrowed and almost obliterated; in others it was par. tially or completely cccluded at some point and dilated on the distal side of the obstruction, occasionally forming when the occlusion was complete a cystic cavity of some dimensions. In many instances it was considerably shortened, measuring only from 1 in. to $1 \frac{1}{2}$ in. in length, instead of from $3 \mathrm{in.}$ to 4 in., its normal length. It was frequently found to be bent on itself and bound down by adhesions, in one case the tip almost touching the crcal end of the process. The contents of the appendix consisted either of clear mucus or of a mucopurulent fluid; in two cases a hard fæcal concretion was present in its interior, and in another a concretion, which had ulcerated through its wall, was found in an abscess cavity external to it. In most cases the inflammatory ehanges were not confined to the appendix itself, for evidences of appendicular peritonitis were generally found to be present, the peritonitis being usually of the adhesive character, the inflammatory exudation which had been poured out round the appendix having undergone organisation and forming adhesions. These were often very firm and extensive, surrounding the appendix and fixing it to the parietal peritoneum, omentum, cæcum, or small intestine. In some instances they were present after a second attack, as in Case 5 ; in other instances, they were absent after many attacks, as in Cases 8 and 15, where the appendix did not show the slightest trace of an adhesion. When exceptionally dense and extensive complete obstruction of the botwels may be produced from inclusion and compression of a coil of small intestine in the adhesions, and if the condition is incapable of relief by operation the result is necessarily fatal. This happened in Case 18, the attack, which was the third, being accompanied by all the symptoms of acute obstruction, which was the predominant feature in the case. On opening the abdomen the appendi $x$ and cæcum were found surrounded by an extensive mass of dense adhesions, included in which were several coils of small intestine, so firmly and so intricately matted together that it was quite impossible to liberate them. In some instances the peritonitis was of the suppurative character, pus having formed in the neighbourhood of the appendix. This complication was met with in

A communication read before the Manchester Pathological Societ on April 14th, 1897 . . 